Job et Samson : hybridation des mythes dans le recueil Hiob oder die Vier Spiegel de Karl Wolfskehl (1869-1948)

Hiob und Simson: Hybridität der Mythen in Hiob oder die Vier Spiegel von Karl Wolfskehl (1869-1948)

Job and Samson: Hybridisation of the Myths in Karl Wolfskehls (1869-1948)

Poem Hiob oder die Vier Spiegel

\title{
Sonia Schott
}

\section{(Q) OpenEdition}

\section{Journals}

Édition électronique

URL : http://journals.openedition.org/ceg/4549

DOI : $10.4000 /$ ceg. 4549

ISSN : 2605-8359

Éditeur

Presses Universitaires de Provence

Édition imprimée

Date de publication : 2 mai 2019

Pagination : 137-150

ISBN : 979-10-320-0214-8

ISSN : 0751-4239

\section{Référence électronique}

Sonia Schott, « Job et Samson : hybridation des mythes dans le recueil Hiob oder die Vier Spiegel de Karl Wolfskehl (1869-1948) », Cahiers d'Études Germaniques [En ligne], 76 | 2019, mis en ligne le 02 novembre 2019, consulté le 25 janvier 2021. URL : http://journals.openedition.org/ceg/4549; DOI : https://doi.org/10.4000/ceg.4549 


\section{Job et Samson : hybridation des mythes dans le recueil Hiob oder die Vier Spiegel de Karl Wolfskehl (1869-1948)}

Sonia SCHOTT

Université Paul-Valéry Montpellier 3 - Université Toulouse-Jean Jaurès, CREG (EA 4151)

Né en 1869 à Darmstadt dans une famille juive aisée, Karl Wolfskehl est l'un des premiers membres du cercle de Stefan George (George-Kreis). Il publie des poèmes et des textes en prose dans Les Feuilles pour l'art (Die Blätter für die Kunst), l'organe de diffusion du mouvement symboliste initié par George. Ses trois recueils de poèmes publiés entre 1897 et $1927^{1}$ s'inscrivent dans des esthétiques Jugendstil, symboliste et expressionniste. Le poète est avant 1933 un personnage important pour la vie culturelle munichoise : lors de réceptions hebdomadaires en sa demeure ont lieu non seulement les célébrations solennelles de la parole poétique par le Cercle de George mais aussi des fêtes costumées plus enjouées, dont l'apogée est constituée par les réjouissances du carnaval.

L'année 1933 marque un tournant déterminant pour l'existence et la production de Wolfskehl : son mentor Stefan George décède et le NSDAP emporte les suffrages en Allemagne. Juste après l'arrivée d'Hitler au pouvoir, Wolfskehl part en exil pour l'Italie. Après que le régime de Mussolini s'est aligné sur l'antisémitisme nazi, le poète se voit contraint en 1938 de s'exiler en Nouvelle-Zélande où il demeure jusqu'à sa mort en 1948. L'exil et le triomphe du nazisme suscitent cependant chez Wolfskehl un renouveau poétique, lié à la profonde crise existentielle que traverse l'auteur. Il n'hésite pas à dire qu'il se considère, dans le sillage de Dante, " a patria pulsus exul immeritus ». Ses œuvres d'exil peuvent être caractérisées par trois notions essentielles que nous développons brièvement.

Le déchirement transparaît dans le poème An die Deutschen où le poète essaie coûte que coûte de maintenir l'impossible unité entre sa « germanité » et « judéité ».

La nostalgie imprègne également l'écriture de Wolfskehl. Celle-ci transparaît notamment au sein du cycle Mittelmeer oder die fünf Fenster ${ }^{2}$ dans lequel l'auteur, depuis la lointaine Nouvelle-Zélande, célèbre le monde méditerranéen et la culture gréco-latine. Il puise ce faisant bien évidemment dans le très riche creuset de la mythologie occidentale : les cinq héros paradigmatiques que célèbre le prélude sont

1. Karl Wolfskehl, ULAIS, Berlin, Bondi Verlag, Blätter für die Kunst, 1897; Gesammelte Dichtungen, ibid., 1903 ; Der Umkreis, ibid., 1927.

2. Ibid., p. 178-193. 
Prométhée, Héraclès, Job, le Christ et Dionysos. En outre, la Magna Mater règne en maîtresse absolue sur les cinq poèmes-fenêtres qui s'ensuivent, ce qui n'est guère étonnant quand on sait que Wolfskehl était un fervent lecteur de Bachofen.

La redécouverte du judaïsme ou l'affirmation de sa judéité constitue la troisième caractéristique de l'œuvre d'exil de Wolfskehl. Fidèle à la figure du poeta vates hérité d'Hölderlin et à la tradition prophétique des Nebiim, le poète retrace l'histoire du peuple d'Israël et s'interroge quant à sa destinée au sein du cycle Die Stimme spricht $^{3}$ sous forme d'un dialogue avec YHWH. Le recueil quadripartite INRI ${ }^{4}$ problématise en outre la reconnaissance de la messianité du Christ et affirme à cet égard la singularité du peuple juif. Et l'œuvre Hiob oder die vier Spiegel porte en son titre l'inscription de l'auteur dans la tradition vétérotestamentaire.

\section{L'ambivalence de Wolfskehl dans son rapport au mythe}

Le mythe (grec, nordique, juif) est un élément essentiel de la pensée et de la poétique wolfskehlienne. Les mythologèmes qui font l'objet de cet article ne constituent en rien une exception au sein de ses œuvres complètes. Poeta doctus et poeta vates, Wolfskehl est le tenant d'une conception ambivalente, voire paradoxale du mythe.

Dans la poétique wolfskehlienne, le mythe est d'une part le médium d'une représentation esthétique. Poeta doctissimus, Wolfskehl compte en effet parmi les membres les plus cultivés du Cercle de Stefan George : certains de ses poèmes qui traitent de figures mythiques sont des Rollengedichte qui témoignent de l'érudition de l'auteur. On peut affirmer alors avec Cornelia Blasberg, qui qualifie cet usage de masque langagier (Sprachmaske), que le but d'un tel recours au mythe obéit au credo symboliste : il s'agit, par le biais de l'anachronisme même que constitue la convocation du mythe, de rendre le lecteur attentif à la langue en elle-même, de susciter une prise de distance par rapport à l'énoncé ${ }^{5}$. Eu égard à la richesse et à la diversité des mythes que l'on trouve dans les poèmes de jeunesse de Wolfskehl, on peut affirmer avec Volker Mertens ${ }^{6}$ et Julia Zernack ${ }^{7}$ que le syncrétisme de Wolfskehl est typique de la modernité poétique.

Le corollaire de cette attitude savante est l'exaltation utopique d'une présence effective du mythe par Wolfskehl qui se considère également comme poète prophète ou poète voyant, poeta vates. Rappelons à cet égard que, de 1899 à 1904, l'auteur était l'un des piliers (avec Ludwig Klages et Alfred Schuler) du mouvement des

3. Ibid., p. 127-172.

4. Ibid., p. 194-202.

5. Cornelia Blasberg, "Weißer Mythos und schwarze Feste. Karl Wolfskehls Antikerezeption », in Achim Aurnhammer, Thomas Pittrof (éd.), „,Mehr Dionysos als Apoll“. Antiklassizistische AntikeRezeption um 1900, Frankfurt a. M., Klostermann, 2002, p. 445-470. [cit. p. 454]

6. Volker Mertens, «Fern-Nähe. Ältere deutsche Literatur und Mythologie im Werk Wolfskehls », in Elke-Vera Kotowski, Gert Mattenklott (éd.), «O dürft ich Stimme sein, das Volk zu rütteln! ». Leben und Werk von Karl Wolfskehl (1869 -1948), Hildesheim/ Zürich/ New York, Olms, 2007, p. 133-148.

7. Julia Zernack, « Nordische Mythen in der deutschen Literatur. Eddaspuren bei Stefan George und Karl Wolfskehl », in Annette Simonis (éd.), Intermedialität und Kulturaustausch. Beobachtungen im Spannungsfeld von Künsten und Medien, Bielefeld, transcript Verlag, 2009, p. 19-41. 
Cosmiques munichois. Dans ses textes en prose, Wolfskehl lui-même forge divers mythes ou élabore une pensée mythique : ainsi Der Priester vom Geiste ${ }^{8}$ et Über die Dunkelheit ${ }^{9}$ développent l'idée d'une réalité plurielle. Le monde serait selon Wolfskehl constitué de plusieurs couches, et selon cette vision nettement teintée d'ésotérisme, le monde visible et tangible n'est qu'une strate qui voile une existence plus profonde, originelle et nocturne. De même, dans un essai publié dans les années 1920, Geist und Gegenwart, Wolfskehl soutient que le poète peut faire «l'expérience d'une communion hors du temps avec un passé devenu quasiment divin, fruit d'une apothéose ${ }^{10} »$. Une semblable idée se trouve développée dans Über historische Treue $^{11}$ qui traite d'une " force sombre et attirante qui [...], venant du lointain et surpassant le lointain, unit l'ici et le maintenant avec le passé et le lointain ${ }^{12}$ ». L'essai Überlieferung professe de façon encore plus explicite que « chaque ère peut faire place à un mythe qui devient réalité ${ }^{13} »$.

Ce riche terreau spéculatif et spirituel constitue les bases sur lesquelles se forge l'œuvre de maturité du poète, dont fait partie Hiob oder die Vier Spiegel ${ }^{14}$. Le poète a commencé à esquisser ce recueil dès 1938 ou 1939 et y a travaillé quasiment jusqu'à la fin de sa vie ${ }^{15}$.

\section{Hiob oder Die Vier Spiegel. Une brève présentation}

Dans Hiob oder die vier Spiegel, l'un des derniers recueils écrits par Wolfskehl, l'hybridation des mythes est le principe poétologique présidant à l'élaboration de chacun des quatre poèmes-miroirs. Ainsi le mythe de Job est-il combiné à celui d'Israël (Erster Spiegel : Hiob Israel), celui de Samson (Zweiter Spiegel : Hiob Simson), celui du prophète (Dritter Spiegel : Hiob Nabi) et celui du Messie (Vierter Spiegel : Hiob Maschiach).

«Métamorphose tétralogique qui permet d'aboutir au Messie à partir des larmes ${ }^{16}$ » selon André Neher, le recueil s'ouvre sur un prélude qui montre que l'âme, à force d'être vendangée par la douleur, l'amertume et les pleurs, devient peu à peu un miroir de YHWH. L'épreuve de la souffrance doit permettre à l'âme de devenir

8. Karl Wolfskehl, Gesammelte Werke, éd. par Margot Ruben et Claus Victor Bock, vol. II, Übertragungen, Prosa, Hamburg, Claassen Verlag, 1960, p. 184-186.

9. Ibid., p. 186-188.

10. « Erlebnis der ausserzeitlichen Kommunion mit dem gewissermassen göttlich gewordenen, apotheosierten Gleichstufigen aus der Vergangenheit. », ibid., p. 366.

11. Ibid., p. 383-389.

12. « dunkle, ziehende Gewalt, die [...] von der Ferne gestachelt und getrieben und die Ferne überbrückend, das Nun und Hier mit Vergangenem und Entlegenem verbindet », ibid., p. 384.

13. Ibid., p. 395.

14. Première publication du recueil, tiré à part : Hamburg, Claassen, 1950. Puis dans Wolfskehl, Gesammelte Werke, vol. I, p. 203-215.

15. Pour une genèse plus précise de l'œuvre, nous renvoyons à l'ouvrage de Friedrich Voit, Karl Wolfskehl. Leben und Werk im Exil, Göttingen, Wallstein Verlag, 2005, p. 476-484.

16. André Neher, L'identité juive, Paris, Payot, 1977, p. 199. 
imago dei, à l'instar de l'Adam Kadmon ${ }^{17}$ de la kabbale lourianique. Si l'on file la métaphore du miroir, chacun des quatre poèmes est donc à la fois un reflet de l'âme ou une réflexion sur la souffrance humaine.

Le premier poème-miroir, Hiob Israel, se réfère à l'étymologie hébraïque du nom Israel de Genèse 32, $29{ }^{18}$ et consiste en un ample tableau de l'histoire du peuple juif, ce peuple «à la nuque raide » qui ne cesse de briser et de renouveler son alliance avec Dieu. Ce tableau panoptique contient déjà en miniature l'histoire de Samson. Le deuxième miroir, Hiob Simson, se concentre uniquement sur le personnage éponyme. Le texte oscille entre le genre du récit et celui du drame. Il constitue une variation sur le récit du Livre des Juges ${ }^{19}$ où est narrée la mort de Samson torturé par ses geôliers, les Philistins.

Le troisième poème est intitulé Hiob Nabi, ce dernier terme signifiant « prophète » en hébreu. Le poème est constitué d'un dialogue très angoissant entre d'une part le prophète et de l'autre une foule ou un chœur à la manière des tragédies antiques. Conformément au topos de la représentation du prophète ${ }^{20}$, ce dernier vient annoncer une nouvelle que le peuple se refuse à entendre. Le poème de Wolfskehl aborde également une autre facette traditionnelle de la prophétie biblique, puisque le nabi invective et fustige ses contemporains qui refusent de l'écouter. Notre interprétation de ce dialogue rédigé pendant les années 1940 est que l'horrible prophétie, qui n'est finalement pas révélée, relève de l'indicible et telle une Hiobsbotschaft terrible à l'extrême, se réfère à la Shoah.

Après l'échec de la prophétie, Hiob Maschiach, le dernier poème, est celui qui se rapproche le plus de la poésie mystique traditionnelle car il consiste en une (re)connaissance ou illumination graduelle. Le poète reprend essentiellement des éléments de la liturgie pascale juive (Pessah) pour montrer la transfiguration de Job en Messie. Le texte se clôt sur un jeu de pronoms qui ressemble à une écholalie primitive. Le principe à l'œuvre dans ce poème est celui de deux miroirs qui se reflètent l'un l'autre : c'est à la fois Dieu qui s'abaisse en chaque homme ou chaque créature et c'est chaque créature qui brise les frontières de son moi pour s'élever vers Dieu ou en Dieu, ce dont on peut proposer une interprétation lourianique ou hassidique.

Wohl, du kommst zu Dir.

Wohl, du wirst Du.

Aufgang rundum, alles ein Ja-Hallelu. ]...[

Hiob-Du. Maschiach-Du.

17. Gershom Scholem, La kabbale et sa symbolique, traduction de Jean Boesse, Paris, Payot, 1966, p. 174-176.

18. «Dieu dit “On ne t'appellera plus Jacob, mais Israël, car tu as lutté avec Dieu et avec les hommes et

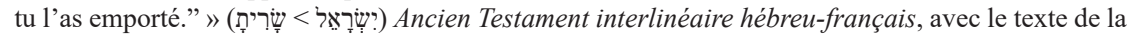
Traduction œcuménique de la Bible et de la Bible en français courant, édition revue et mise à jour, Alliance biblique universelle, Tours, APS Chromostyle, 2011, p. 106. [nous citons la traduction de la TOB.]

19. Juges : 16, 25-30. Ibid., p. 849-850.

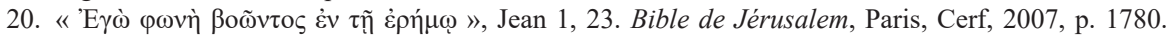
«Moi? la voix de celui qui crie dans le désert [...]. Comme a dit Isaïe le prophète. » 


\section{WER-ER-DU ${ }^{21}$.}

\section{Job et Samson : deux esquisses}

De l'histoire de Job, la tradition a retenu deux interprétations, que Georg Langenhorst résume dans l'intitulé « Ijob - Vorbild in Demut und Rebellion ${ }^{22}$ ». Job incarne en effet l'humilité du juste qui souffre, mais demeure fidèle à Dieu. Cependant, les reproches qu'il adresse à YHWH le présentent également comme une figure prométhéenne, celle de l'homme révolté qui ose remettre en cause la théodicée en demandant s'il y a une raison à la souffrance.

Samson est élu par Dieu pour être son serviteur, son nazir (נזיר.) Dieu lui confère une force herculéenne, ce en quoi le héros biblique s'apparente à une figure de « surhomme ». Ses cheveux qu'ils ne coupe jamais sont le signe de son naziréat et symbolisent l'origine de sa puissance. Les Philistins, ennemis du peuple juif, persuadent Dalila de séduire Samson afin de savoir d'où lui vient sa puissance. La mission de Dalila accomplie, Samson est capturé par les Philistins, qui le font prisonnier et le mutilent en le rendant aveugle. Les poèmes de Wolfskehl consacrés à Samson évoquent sa mort. Le chapitre 16 du Livre des Juges en constitue l'intertexte vétérotestamentaire.

\footnotetext{
Les Philistins le saisirent et lui crevèrent les yeux : ils le firent descendre à Gaza et le lièrent avec une double chaîne de bronze. Samson tournait la meule dans sa prison. [...] Et comme leur cœur était en joie, ils s'écrièrent : «Faites venir Samson pour qu'il nous amuse !» On fit donc venir Samson de la prison et il fit des jeux devant eux, puis on le plaça debout entre les colonnes. Samson dit alors au jeune garçon qui le menait par la main : " Conduis-moi et fais-moi toucher les colonnes sur lesquelles repose le temple, afin que je m'y appuie. Or le temple était rempli d'hommes et de femmes. Il y avait là tous les princes des Philistins, et, sur la terrasse, environ trois mille hommes et femmes qui regardaient les jeux de Samson. Samson invoqua Yahvé et il s'écria : « Seigneur Yahvé je t'en prie, souviens-toi de moi, donne-moi des forces encore cette fois, et que d'un seul coup je me venge des Philistins pour mes deux yeux. » Et Samson tâta les deux colonnes du milieu sur lesquelles reposait le temple, il s'arc-bouta contre elles, contre l'une avec son bras droit, contre l'autre avec son bras gauche et il s'écria : «Que je meure avec les Philistins! » Il poussa de toutes ses forces et le temple s'écroula sur les princes et sur tout le peuple qui se trouvait là. Ceux qu'il fit mourir en mourant furent plus nombreux que ceux qu'il avait fait mourir pendant sa vie ${ }^{23}$.
}

Le titre Hiob oder die Vier Spiegel montre que le personnage éponyme est le point focal de l'ensemble du recueil. Le mythe de Samson est abordé brièvement dans le premier poème-miroir, mais essentiellement dans le deuxième. Samson apparaît en outre dans la seconde version de Die Stimme spricht ${ }^{24}$ au sein du poème « Kalon bekawod namir ».

21. Ibid., p. 215.

22. Georg Langenhorst, « Ijob - Vorbild in Demut und Rebellion », in Heinrich Schmidinger (éd.), Die Bibel in der deutschsprachigen Literatur des 20. Jahrhunderts, vol. II, Mainz, Matthias Grünewald Verlag, 1999, p. 259-280.

23. Juges, 16, 21-30. Ancien Testament interlinéaire, p. 849-850.

24. Wolfskehl, Gesammelte Werke, vol. I, p. 157-158. [Die Stimme spricht] 
« Hiob Simson » est composé de deux parties nettement distinctes. Dans la première partie qui constitue la scène d'exposition du drame de Samson, Wolfskehl ne s'éloigne guère de la source vétérotestamentaire. Son poème s'apparente néanmoins à un commentaire midraschique dans la mesure où il aborde l'action non seulement sur le mode de la focalisation externe, mais également du point de vue interne à Samson. Nous proposons d'assimiler les Philistins, le peuple ennemi du peuple juif, au peuple allemand gangréné par le nazisme, dans une lecture symbolique que semble nous permettre le contexte de rédaction de l'œuvre. Deux autres variations méritent néanmoins d'être prises en compte : alors que dans le Tanakh les Philistins exigent de Samson qu'il fasse des « jeux », dans le poème wolfskehlien, ses ennemis exigent de lui qu'il chante et joue du kinnor. Par ailleurs, les dernières exclamations de Samson dans le récit biblique sont plutôt courtes : elles consistent en deux phrases. En revanche, Wolfskehl enrichit le texte source par une tirade longue de 36 vers qui constitue la seconde partie du poème : le héros torturé se compare à un pin des Landes affligé par la marée et le vent. La richesse littéraire de la tirade se mesure au flou délibéré de la configuration interpellative : cette seconde partie relève en effet conjointement d'un monologue désespéré, d'une accusation envers les Philistins ainsi que d'un cri adressé à YHWH.

Eu égard à la richesse et à la plasticité des mythes bibliques, nous soulignerons quels aspects ou quelle exégèse de chacun des personnages Wolfskehl privilégie. Notre analyse montrera en outre dans quelle mesure les références sémantiques à " des hommes de douleur » ou des martyrs tels que Job et Samson permettent de caractériser le pathos inhérent à l'écriture du poète en mettant en lumière les processus d'hybridation des deux mythes. Nous aborderons la réactualisation wolfskehlienne des mythes en abordant successivement des perspectives identitaire, poétologique et polémique.

\section{Job Samson dans une perspective identitaire}

Dans une lettre ouverte envoyée le 13.9.1946 à son ami Kurt Frenert, le poète condense la fonction identitaire du mythe de Job à la fois sur le plan personnel et sur le plan collectif, au nom du peuple juif :

Vom Tag ab, als das Schiff vom Hafen Europas abstieß, hab ich's gewusst, gelebt, ausgesprochen, ausgeschluchzt, ausgesungen, das Zeichen, unter dem mein Leben, die letzte Phase dieses Erdengangs seitdem steht. Dieses Zeichen, mehr als ein Bild, es ist der ewige Fug des Judenschicksals. Und ich, zuckend und fast widerstrebend gehorsam, fühl ich, der Mitwalter, der Mithüter des deutschen Geistes, ich mich dazu bestimmt, das lebendige, ja das schaffende Symbol dieses Schicksals darzustellen. Seit jenem Augenblick steht alles, was ich bin, was ich füge, unter dem ewigen Namen Hiob, seitdem bin ich, leb ich, erfahr ich Hiob. Alles, was seitdem entstand, führte diesen Namen, oder, wo es abseits gewachsen scheint, ist es von ihm durchweht ${ }^{25}$.

25. Karl Wolfskehl à Kurt Frenert, 13 septembre 1946, in Karl Wolfskehl, Gedichte. Essays. Briefe, éd. par Cornelia Blasberg et Paul Hoffmann, Frankfurt a. M., Jüdischer Verlag im Suhrkamp Verlag, 1999, p.189-194. [Citation p. 192.] 
On remarque à la mise en valeur du champ lexico-sémantique de l'expérience vécue (« leben », « erfahren ») que le mythe de Job est vraiment pour Wolfskehl quelque chose qui se vit et qu'il pense incarner. Cela rappelle de façon ostensible les conceptions ésotériques du mythe qu'il développait dans ses écrits en prose évoqués supra. Bien que cela reste implicite dans la lettre, on peut aisément déduire du contexte historique de la Seconde Guerre mondiale, de l'exil et de la Shoah que Job incarne ici l'homme qui souffre sans savoir pourquoi.

On retrouve l'interrogation existentielle quant à la souffrance humaine dans le cri jobien de Samson qui se compare au conifère malmené par les forces de la nature :

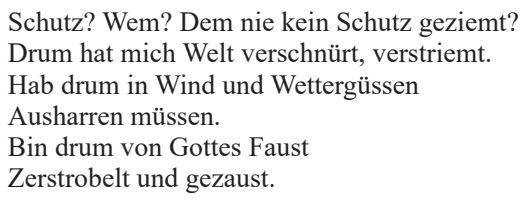

Il va de soi que le cri de détresse du héros est suscité par les tortures auxquelles l'exposent les Philistins. Pour autant, sa vindicte ne s'adresse pas forcément à ces derniers, mais consiste davantage en une accusation voire un cri de fustigation terrible contre Dieu lui-même, à l'instar des passages les plus rebelles du Livre de Job, comme en témoigne l'extrait suivant :

Schrecken stürzen auf mich ein verjagt wie vom Wind ist mein Adel, wie eine Wolke entschwand mein Heil. Und nun zerfließt die Seele in mir, des Elends Tage packen mich an. Des Nachts durchbohrt es mir die Knochen mein nagender Schmerz kommt nicht zur Ruh. Mit Allgewalt packt er mich am Kleid, schnürt wie der Gürtel des Rocks mich ein. Er warf mich in den Lehm, sodass ich Staub und Asche gleiche. Ich schreie zu dir und du erwiderst mir nicht; ich stehe da; doch du achtest nicht auf mich. Du wandelst dich zum grausamen Feind gegen mich, mit deiner starken Hand befehdest du mich ${ }^{26}$.

On ne saurait en effet ignorer la parenté entre la manière dont Job évoque la violence divine dans l'Ancien Testament et les vers wolfskehliens « Bin drum von Gottes Faust/ Zerstrobelt und gezaust ». Il subsiste néanmoins une différence entre les deux personnages : dans le texte biblique, Job a l'audace de s'adresser directement à la deuxième personne au Dieu dont il estime être le martyr; dans le poème de Wolfskehl, l'accusation est moins directe, puisque qu'elle se fait par le recours à l'énallage : Samson parle des maux qu'il endure à la troisième personne.

L'extrême fragilité de l'homme malmené par son Dieu constitue un topos du Livre de Job, qui se manifeste dans le poème d'un point de vue stylistique par le biais de ce qu'Hugo Friedrich ${ }^{27}$ appelle « l'aspect agressivement dramatique [de la poésie moderne, $n d l a$.]». Les derniers vers de la tirade du héros tendent vers la désarticulation de la syntaxe. On ne peut par ailleurs identifier aucune régularité dans le rythme de l'ensemble de la tirade, ce qui contribue également à renforcer l'impression de chaos.

26. Ijob, 30, 15-21. Die Bibel, Einheitsübersetzung, Freiburg/ Basel/ Wien, Herder, 2005, p. 602-663.

27. Hugo Friedrich, Structure de la poésie moderne. Traduit de l'allemand par Michel-François Demet, Librairie générale française, 1999, p.17. 
Ich Hüter brech euch selbst die Bahn:

Heran! Heran!

Brünstiger Untergang

Gottes: vorbei!

Drang - Drang -

Dans sa lettre à K. Frenert, Wolfskehl s'identifie par un pacte lyrique tacite au personnage de Job et conclut également ce pacte au nom de l'ensemble du peuple juif. L'hybridation des mythes de Job et de Samson recouvre alors une fonction identitaire pour l'auteur et tous les Juifs confrontés au chaos de l'exil, des pogroms et de la Shoah. La seule identité qui les caractérise demeure un destin d'une absolue précarité située aux limites de l'absurde.

\section{Job Samson dans une perspective poétologique}

Dans une lettre à son ami Abraham Scholem Yahuda ${ }^{28}$, le douloureux paradoxe que souligne Wolfskehl consiste en un martyre poétologique : rejeté par les Allemands, il n'a que la langue allemande comme refugium poétique :

Ein deutscher Dichter zu sein ist für den heutigen deutschen Juden wohl die schwerste Prüfung und Erprobung - nach beiden Seiten! Freilich bin ich nicht der Einzige, den dies Fatum lebendig betrifft. Dass ich hierbei gar nichts Äusserliches im Auge habe, dafür kennen Sie mich. Das Äusserliche wäre zu bewältigen oder, wird es stärker als wir, dann mag es uns verschütten, dann waren wir nicht mehr wert. Aber die innere Situation! Ich habe nichts als das deutsche Wort, ich habe es als Forderung wie als Besitz, als Antrieb und Mittel und als die geheimnisvolle Quelle meiner Kraft. Und doch: der Ruf ist in mir laut geworden, die Stimme sprach und sie sprach als deutsches Wort, sie spricht und spricht weiter.

La dramaturgie de la première partie de «Hiob Simson » retrace ce paradoxe :

Sie liessen ihn aus den Verliesen holen,

Sein Leid als köstlichen Rauschtrank auszuschlürfen.

Er solle, höhnten sie, wieder singen dürfen,

Wie droben im Gebirg, wo Lenz den Talen

Sein Lied vorbraust in der Brunst wild seufzender Qualen.

« Spiel auf! hier, greif dein Kinnor, sing, wir lauschen! »

Er aber stand entfesselt auf den Bohlen,

Ihm wars, er höre Meeresflut herüberrauschen.

Und stünd allein im flachen Muschelstrand.

« Halt dich am Pfeiler »-schrien sie. Rote Scham

Dass er gehorche, den Blinden überkam.

Die Lippe nagt er und die Schläfe fliegt

Vorm johlenden Saal, dumpf keuchend, nackentief

Das schwere Lockenhaupt, wie wenn er schlief,

Nichts hinterm Auglid. Knie schlägt an Knie.

«Tu deinen Dienst, Hand! » - schluchzt er auf, verkrampft.

Ihm schien es, dass ein Egel ihm entzieh

Den letzten Tropfen, drin noch Leben dampft,

Zuckendes Leben. « Nun, was singt er nicht?

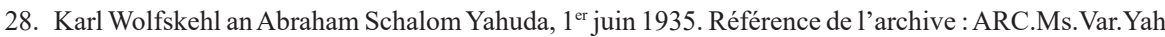
38013034.13 Abraham Schalom. Wolfskehl Karl, National Library of Israel, Hebrew University, Jérusalem. 


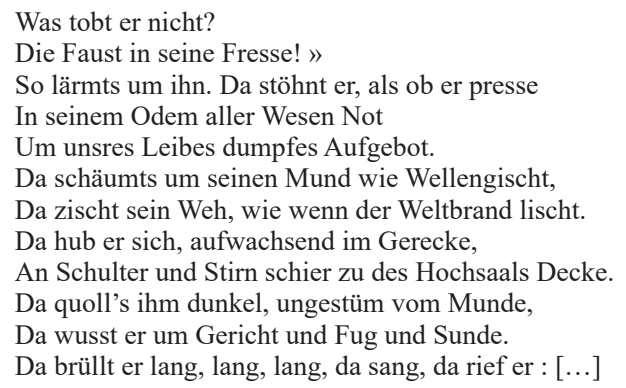

On ne peut passer outre le lien intertextuel qui rapproche ce dernier extrait de celui du Psaume 137 et de Todesfuge de Paul Celan. Dans chacun des cas, les oppresseurs demandent aux opprimés de chanter ou de jouer de la musique. Au regard des rapports de force, de la violence des Philistins, des Babyloniens et du soldat allemand/ nazi, le chant et la musique ne relèvent plus de l'art mais de la torture pour ses exécutants :

Là-bas, au bord des fleuves de Babylone, nous restions assis tout éplorés en pensant à Sion. Aux saules du voisinage nous avions pendus nos lyres. Là, nos conquérants nous ont demandé des chansons, et nos ravisseurs des airs joyeux : «Chantez-nous quelque chant de Sion. » Comment chanter un chant du Seigneur en terre étrangère? Si je t'oublie Jérusalem, que ma droite oublie ${ }^{29} \ldots$ !

L'imbrication entre torture et musique est renforcée de façon encore plus intime dans le poème de Celan, qui mêle inextricablement les évocations des meurtres et les cris ordonnant de chanter et de jouer de la musique :

29. Ancien Testament interlinéaire, p. 2161-2162. Psaume 137. [C'est nous qui soulignons, ndla.] 
Todesfuge ${ }^{30}$

$[\ldots]$

er pfeift seine Juden hervor läßt schaufeln ein Grab in der Erde er befiehlt uns spielt auf nun zum Tanz

$[\ldots]$

Er ruft stecht tiefer ins Erdreich ihr einen ihr andern singet und spielt er greift nach dem Eisen im Gurt er schwingts seine Augen sind blau stecht tiefer die Spaten ihr einen ihr andern spielt weiter zum Tanz auf

$[\ldots]$

Er ruft spielt süßer den Tod der Tod ist ein Meister aus Deutschland er ruft streicht dunkler die Geigen dann steigt ihr als Rauch in die Luft dann habt ihr ein Grab in den Wolken da liegt man nicht eng

Dans le poème de Wolfskehl, différents procédés manifestent la torture de Samson : l'opprobre subi («Rote Scham ») est mis en valeur par le contre-rejet, tandis que la personnification et le bouleversement syntaxique rendent sensible au lecteur la honte qui envahit Samson. Au treizième vers « Die Lippe nagt er und die Schläfe fliegt », l'antéposition du complément d'objet direct souligne le déchirement intérieur et le vœu de mutisme de Samson. C'est encore dans la gestuelle du héros que l'on devine la peur quand l'attention se concentre sur les jambes du héros quelques vers plus loin : «Knie schlägt an Knie ». L'acmé de la division du héros est atteinte par l'introduction du discours direct : comme séparé de lui-même, Samson doit ordonner à sa propre main de jouer : «Tu deinen Dienst, Hand! » - schluchzt er auf, verkrampft. » Il n'y parvient toutefois pas et les Philistins le menacent encore davantage; il pousse ensuite un long hurlement, qui constitue la seconde partie $\mathrm{du}$ poème.

Le vers 32, qui introduit pour la seconde fois le discours direct, met sur le même plan l'action de hurler (« brüllen »), chanter (« singen ») et crier (« rufen ») : « Da brüllt er lang, lang, lang, da sang, da rief er ». D’un point de vue poétologique, tout ici donne l'impression que la parole poétique des Juifs martyrisés et du poète en exil qui leur prête voix ne peut plus consister qu'en un long cri. L'association entre ces trois verbes consacre, selon la formule de Nathalie Vincent-Arnaud, le cri comme « archétype d'une révolte de tout l'être face à la violence perpétrée sur l'humain ${ }^{31}$ ». La révolte s'exprime au travers des questions rhétoriques, dont la teneur est renforcée dans les premiers vers par l'épiphore (" mich Dünenkiefer ») ainsi que par l'enjambement qui par deux fois brise le vers pour mettre en valeur le verbe « müssen ». La révolte de Samson est motivée avant tout par son incompréhension de la violence à laquelle il exposé. Force est alors de reconnaître également l'infinie

30. Paul Celan, Choix de poèmes réunis par l'auteur. Traduction et présentation par Jean-Pierre Lefebvre. Édition bilingue, Paris, Gallimard, 1998, p. 53-57.

31. Nathalie Vincent-Arnaud, "Corps, chœurs et cris : les voix de la guerre dans trois œuvres contemporaines », in Valérie Morisson (éd.), Le Cri dans les arts et la littérature, Dijon, Éditions Universitaires de Dijon, 2017, p. 187-195. [cit. p. 189.] 
vulnérabilité du héros, reflétée par sa question désespérée « Schutz? Wem? Dem nie kein Schutz geziemt? » et par le constat de son dénuement : ce dernier est symbolisé par la dégradation du pin rongé par les vers et dont les branches sont nues, ce que la rime plate assez désagréable à entendre contribue à rendre sensible :

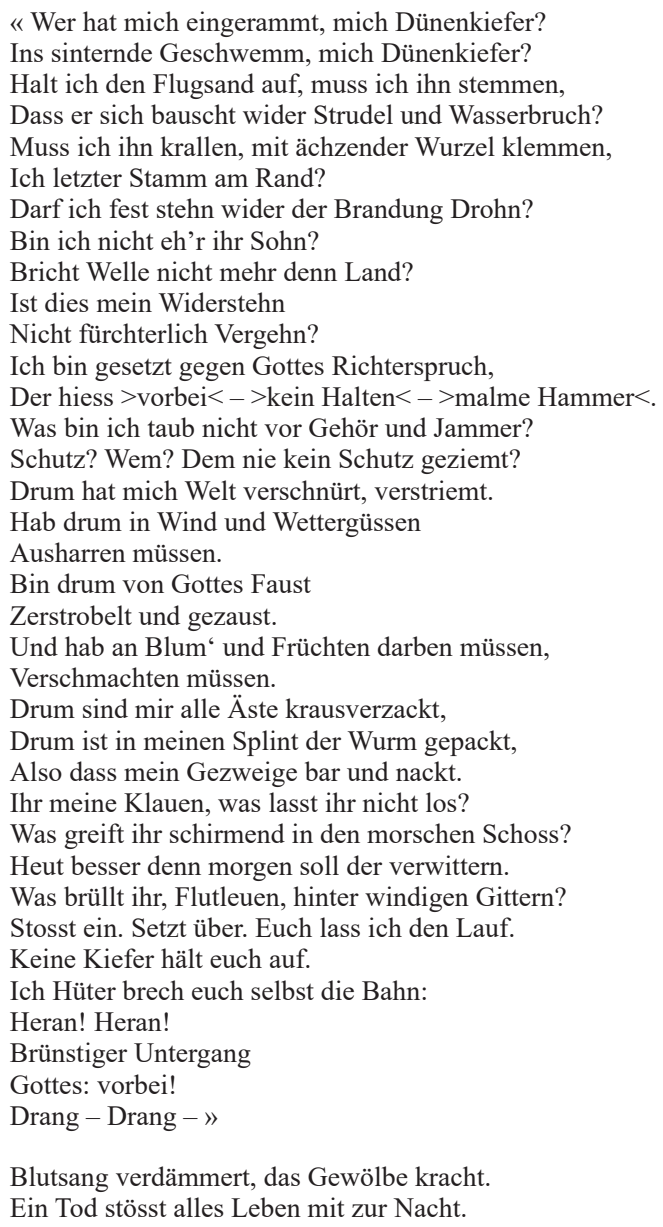

Blutsang verdämmert, das Gewölbe kracht.

Ein Tod stösst alles Leben mit zur Nacht.

Le cri que pousse Samson est à la fois un cri de détresse immense mais il relève également de la vindicte. Si nous venons de démontrer que l'un des destinataires peut en être Dieu lui-même, le cri du héros peut néanmoins également être dirigé contre ses geôliers. La vengeance de l'opprimé est en effet terrible, qui procède à la fois de l'autodestruction et du meurtre des ennemis. Le mythe de Wolfskehl s'inscrit ce faisant dans une perspective polémique. 


\title{
Job Samson dans une perspective polémique
}

Samson apparaît également au sein du recueil Die Stimme spricht. Ci-après, un extrait de ce dernier recueil où le héros a les traits d'un vengeur :

\author{
Das Makelmal - Ehrenmal. \\ Wir tragens aus Gottes Wahl, \\ Wir tragens als Feiertracht, \\ Wir tragens zur Weiheschlacht, \\ Wir tragens, ein Siegspanier: \\ Kalon bekawod namir! \\ $[\ldots]$ \\ Voraus in fliegendem Haar \\ Ein Blinder, der Rächer war, \\ Und der König, gesalbt vom Herrn, \\ Psalter, Schwert, Stern. \\ Mit euch Helfern, euch Helden, wir: \\ Kalon bekawod namir!
}

Le titre du poème Kalon bekawod namir constitue une référence historique à un mouvement de protestation qui a eu lieu en Israël (alors territoire palestinien sous mandat britannique) : lorsque la population juive locale apprit que ses « frères allemands » devaient porter l'étoile jaune sous la contrainte des nazis, certains Juifs attachèrent alors sur leurs vêtements un insigne jaune ${ }^{32}$. L'Allemagne nazie est représentée ici par le peuple d'Edom, ennemi traditionnel du peuple d'Israël. La traduction et translittération du vers qui cite le slogan inscrit sur l'écusson sont les suivantes :

KALON : קלון : 1'opprobre.

BEKAWOD : בכבוד : en honneur

NAMIR : נמיר : nous changerons.

Le cri de protestation et cette transmutation se traduisent dans le poème wolfskehlien par l'oxymore « das Makelmal-Ehrenmal ». Une telle transfiguration montre encore combien l'hybridation des mythes de Samson et de Job est fructueuse : le martèlement de la volonté de transformer les stigmates en marques d'honneur rappellent évidemment la transfiguration qui s'opère à la fin du Livre de Job, à qui YHWH donne une dignité nouvelle. En revanche, à la différence du personnage de Job qui incarne l'humilité, le martèlement incessant et polémique du vers « Kalon Bekawod Namir » résonne comme une fière malédiction contre les ennemis d'Israël. On soulignera à cet égard que la scansion, les rimes plates qui évoquent un martèlement tout militaire, le choix des mots - qui semblent empruntés à la très germanique Hermannsschlacht de Kleist - ainsi que les rimes très signifiantes entre « triumphier », « Siegspanier » et « namir » revêtent une forme éminemment germanique, quand bien même les héros

32. Des affiches en faveur de ce mouvement et des photos de personnes défilant dans Jérusalem en portant l'insigne sont disponibles dans les archives de le bibliothèque nationale de Jérusalem sous la côte : V 2932\4, NLI, Hebrew University, Jérusalem. 
du combat évoqué seraient chargés d'affirmer leur judaïté et s'inscriraient ce faisant contre les oppresseurs nazis. Ainsi, même dans un poème constituant un véritable cri de guerre et défendant Israël, Wolfskehl ne peut faire cela que dans la langue qui lui est sienne, l'allemand.

De même que la fierté l'emporte sur l'humilité dans le poème précédent, la figure de Job Samson se laisse difficilement apparenter à l'image d'un Job qui incarnerait la soumission. Dans la tirade qu'il débute avant de mourir - ou d'être sacrifié Samson se compare à un pin des Landes. La référence à Théophile Gautier ${ }^{33}$ est plutôt explicite. Ici, la métaphore sylvestre est éminemment tragique car elle montre que le pin des Landes est confronté à des forces bien plus violentes que lui, qui semblent disposer de son destin. La mort de Samson, dont seul le suicide peut sauver l'honneur, évoque la grandeur des figures tragiques, tel l'Ajax sophocléen. Il n'est donc guère surprenant que Franz Rosenzweig ait rangé Samson aux côtés de Gilgamesh et des héros tragiques dans Der Stern der Erlösung ${ }^{34}$. Samson complète donc Job de par la nature belliqueuse et vindicative de son entreprise.

\title{
Conclusion
}

\author{
Gebot dem Blinden war: miss deine Kräfte \\ Die Hand, die weisende, führte die Hand. \\ Mit schwerer Hand abbogst des Hauses Schäfte \\ Dich schlagend schlug dein Gott den Götzentand ${ }^{35}$
}

La seule limite à l'hybridation des deux mythes que nous soulignerons en conclusion est la différence entre le sort réservé à Samson, qui meurt, et Job, qui est sauvé. Dans le poème « Hiob Israel » cité supra, nous remarquons en outre à la suite de Paul Hoffmann ${ }^{36}$ une différence essentielle entre le texte vétérotestamentaire et le midrash qu'en livre Wolfskehl : dans le Livre des Juges, c'est Samson qui, de lui-même, pousse les colonnes du temple afin qu'elles s'effondrent sur lui et les Philistins. En revanche, dans l'interprétation wolfskehlienne, c'est la main de Dieu (« die Hand, die weisende, führte die Hand ») qui pousse Samson à l'autodestruction, ce que confirme quelques vers plus bas le polyptote («schlagend schlug »). Samson apparaît ici comme l'instrument de la colère divine qui, dans un mouvement plutôt démoniaque, ne sait que retourner la violence contre son nazir et contre lui-même pour abattre ses ennemis.

33. Théophile Gautier, Euvres poétiques complètes, éd. établie par Michel Brix, Paris, Bartillat, 2004, p. 371. [《Le Pin des Landes »]

34. Franz Rosenzweig, Der Stern der Erlösung. Mit einer Einführung von Reinhold Mayer und einer Gedenkrede von Gershom Scholem, Frankfurt a. M., Suhrkamp, 1988.

35. «Der erste Spiegel: Hiob Israel », in Wolfskehl, Gesammelte Werke, p. 204, vers 15-18.

36. Paul Hoffmann, Das religiöse Spätwerk Karl Wolfskehls, thèse de doctorat non publiée, soutenue à l'université de Vienne, 1958, p. 124-126. [Le document est disponible dans les archives littéraires de Marbach a. Neckar.] 
L'approche étymologique ${ }^{37}$ montre que les lettres qui constituent le nom de Job en hébreu sont, à une inversion près, les mêmes que celles du mot « ennemi » (איוב / איוב). Cette coïncidence onomastique nous semble symbolique des tensions autodestructrices qui constituent, d'une part, un élément fondamental de la combinaison du mythe de Samson et de celui de Job et elle permet, d'autre part, de caractériser l'inconciliable identité judéo-allemande de Karl Wolfskehl.

Par ailleurs, le nom de Samson provient du nom du soleil (ששְמשוֹן > שמש). Or dans l'œuvre de Wolfskehl, Samson est un personnage obscur et tragique. En effet, le traitement du mythe biblique par Wolfskehl n'est pas sans rappeler le Crépuscule des dieux de Wagner, par le parallèle que l'on peut établir entre la torture du pin chez Wolfskehl et la mutilation du frêne chez Wagner, et surtout par l'effondrement du temple dans le poème et la destruction du Walhalla à la fin de la Tétralogie. Pour autant, la destruction chez Wolfskehl ne s'ouvre pas sur la Rédemption par l'amour comme dans l'œuvre wagnérienne, mais sur le cri angoissé de Hiob Nabi, qui s'élève dans les ruines.

37. C'est notre professeure d'hébreu, Danièle Ellul, qui nous a transmis ce savoir. Qu'elle reçoive ici l'expression de notre gratitude. 